\title{
IRREGULARITIES OF PARTITIONS
}

Ramsey theory, uniform distribution

V.T.Sós

Eötvös Lorănd University, Budapest, Mazeum krt. 6-8, Hungary

\begin{abstract}
In this survey we discuss a common feature of some classical and recent results in number theory, graph theory, etc. We try to point out the fascinating relationship between the theory of uniformly distributed sequences and Ramsey theory by formulating the main results in both fields as statements about certain irregularities of partitions. Our approach leads to some new problems as well.
\end{abstract}

\section{INTRODUCTION}

In 1915 Hermann heyl published his classical paper entitled "Ober die Gleichverteilung von Zahlen mod Eins". This was intended to furnish a deeper understanaing of the results in diophantine approximation and to generalize some basic results in this field. The theory of uniformly distributed sequences has originated with this paper. In the last decades this subject has developed into an elaborate theory related to number theory, geometry, probability theory, ergodic theory, etc.

Curiously enough, Issai Schur's paper entitled "Ober die Kongruenz $x^{n}+y^{n} \equiv z^{n}$ (mod $\left.p\right)$ " appeared in the very same year. He proved that if the positive integers are finitely colored, then there exist $x, y$, $z$ having the same color so that $x+y=z$. Though Ramsey theory has various germs, Schur's theorem can be regarded as the first Ramsey-type theorem. Now literally the same applies to Ramsey theory as to the theory of uniform distribution:

In the last decades Ramsey theory became an elaborate theory related to number theory, geometry, probability theory, ergodic theory, etc.

It took about half a century for both fields to become coherent theories. It took more than a decade to realize the close relationship between the two seemingly unrelated areas. The interaction between the theory of uniform distribution and combinatorics in general is indicated in several works. We list without claiming completeness some of 
them: Erdరs \& Spencer (1972), Graham, Rothschild \& Spencer (1980), olson \& Spencer (1978), Niederreiter (1972), Tijdeman (1980). A breakthrough in this direction has been achieved in a recent series of papers by $\mathrm{J}$. Beck (1981 a, b, c, d), (1983 a, b, c, d).

\section{FORMULATION OF THE GENERAL PROBLEM}

As introductory examples we consider some classical theorems. The first is one of the basic results in the theory of uniform distribution.

Let $x_{1}, \ldots, x_{N}$ be $N$ points in the unit square. Let I be an aligned rectangle, i.e. one with sides parallel to those of the unit square. Denote by $Z$ (I) the number of points $x_{i}, 1 \leq i \leq N$ in $I$. $|I|$ denotes the Lebesgue-measure of I .

Theorem 1.1. (Schmidt (1972)). There exists an aligned rectangle $I_{0}$ such that

$$
\left|Z\left(I_{0}\right)-N\right| I_{0}||>\operatorname{clogN}
$$

holds, where $c>0$ is a positive absolute constant.

Theorem 1.2. (Roth (1964)). Let $[N]=\{1, \ldots, N\}$. For any partition $[i \mathrm{i}]=\mathrm{S}_{1} \cup \mathrm{S}_{2}, \mathrm{~S}_{1} \cap \mathrm{S}_{2}=\emptyset$ there exists an arithmetic progression $P=\{a, a+d, \ldots, a+k d\} \subset[N]$ such that

$$
|| P \cap S_{1}|-| P \cap S_{2}||>C H^{1 / 4}
$$

holds, where $c$ is a positive absolute constant.

Theorem 1.3. (Ramsey (1930)). For $n>n_{0}(t)$ if the edges of $\mathrm{K}_{\mathrm{n}}$ (the complete graph on $\mathrm{n}$ vertices) are 2-colored, then there must be a monochromatic $\mathrm{K}_{\mathrm{t}}$.

The theorems stated above have a common feature. In all three of them we are given an underlying set $S$ and a family of subsets of this set, and (in all three cases) the claim is that the underlying set has no partition which splits proportionally or equally each set contained in the given family.

We now give a formulation of the general problem.

Let $S$ be a set and $A \subseteq 2^{S}$ a family of subsets of $S$. Let $G$ denote the set of functions (partitions, colorings) $g: S \rightarrow\{1, \ldots, r\}$. 
or more generally let us fix real numbers $\alpha_{1}, \ldots, \alpha_{r}$ and let $G$ denote the set of functions $g: S \rightarrow\left\{\alpha_{1}, \ldots, \alpha_{r}\right\}$. We shall use various functions

$$
\Delta: G \times A \rightarrow R
$$

to measure the discrepancy (weighted nonuniformity) $\Delta_{g}(A)$ of geG on A. Given such a discrepancy function, our goal is to estimate the following quantities:

I The discrepancy of $g$ over A :

$$
\Delta_{g}(A)=\sup _{A \in A}\left|\Delta_{g}(A)\right|
$$

or e.g.

$$
\Delta_{g}^{P}(A)=\left(\sum_{A \in A}\left|\Delta_{g}(A)\right|^{P}\right)^{\frac{1}{p}}
$$

and the discrepancy of $G$ over $A$ :

$$
\Delta_{G}(A)=\inf _{g \in G} \Delta_{\underline{g}}(A) .
$$

II The one-sided discrepancies of $g$ over $A$ :

$$
\begin{aligned}
& \Delta_{g}^{+}(A)=\sup _{A \in A} \Delta_{g}(A) \\
& \Delta_{g}^{-}(A)=\left|\inf _{A \in A} \Delta_{g}(A)\right|
\end{aligned}
$$

and

$$
\Delta_{G}^{+}(A)=\inf _{g \in G} \Delta_{g}^{+}(A), \quad \Delta_{G}^{-}(A)=\inf _{g \in G} \Delta_{g}^{-}(A) .
$$

In some cases we consider only partitions of $S$ which satisfy certain requirements. This means that we have a $G^{*} \subseteq G$ and we investigate $\Delta_{G^{*}}(A)=\inf _{g \in G^{*}} \Delta_{g}(A)$. 
Consider the case $r=2$. Here the simplest problem is the fol-

lowing:

We wish to find a two-coloring of $S$ so that every set AEA is partitioned by this into parts of size as equal as possible. Now a two-coloring of $S$ can be given by a function $g: S \rightarrow\{+1,-1\}$. The function $\Delta$ defined by

$$
\Delta_{g}(A)=\sum_{x \in A} g(x)
$$

measures the discrepancy of this partition on $A$.

$$
\Delta(A)=\inf _{g} \sup _{A \in A}\left\{\sum_{x \in A} g(x)\right\}
$$

measures (in supremum normi) how well under the requirement above the set $S$ can be partitioned.

Now consider a more general problem. We want to find a system of representatives of $A$ so that in every set $A \in A$ the number of representatives is proportional to $|A|$, i.e. for a given $\alpha \in(0,1)$ it is as close to $\alpha_{\mid} A \mid$ as possible. This means that we consider partitions of ratio $\alpha, 1-\alpha$. To handle this problem now we take the functions $g: S \rightarrow\{\alpha-1, \alpha\}$ and

$$
\Delta_{g}(A)=\sum_{x \in A} g(x)
$$

As above, define

$$
\Delta(A)=\inf _{g} \sup _{A \in A} \mid \sum_{x \in A} g(x) !
$$

Note that if $g^{-1}(\alpha-1)=s_{1}, g^{-1}(\alpha)=s_{2}$, then

$$
\sum_{x \in A} g(x)=|A| \alpha-\left|S_{1} \cap A\right|
$$

Consequentiy, this measures the discrepancy in an appropriate way.

Let us reformulate the above theorems in this general setting. 
From the proof of Schmidt's theorem it is easy to see that it can be given in the following finite form.

Theorem 1.1*. (the finite version of Schmidt's theorem). Let

$$
\begin{aligned}
& S=\{(i, j) ; 1 \leqq i \leqq N, \quad 1 \leqq j \leqq N\}, \\
& A_{k, \ell}=\{(i, j): 1 \leqq i \leqq k, \quad 1 \leqq j \leqq \ell\}
\end{aligned}
$$

and

$$
A=\left\{A_{k, l}: 1 \leqq k \leqq N, \quad 1 \leqq \ell \leqq N\right\}
$$

Set

$$
G=\left\{g: S \rightarrow\left\{1-\frac{1}{N},-\frac{1}{N}\right\}\right\}
$$

and $\Delta_{g}(A)=\sum_{x \in A} g(x)$.

Then

$$
\Delta_{G}(A) \geqq c \log N
$$

where $c$ is a positive absolute constant.

Further, Roth's theorem can be formulated in an obvious way as follows.

Theorem 1.2*. (Roth). Let $S=[N], A=\{A: A \subseteq S, A$ is an arithmetic progression $\}, G=\{g: N \rightarrow\{+1,-1\}\}$. Put $\triangle_{g}(A)=$ $=\left|\sum_{x \in A} g(x)\right|$. Then

$$
\Delta_{G}(A)>\mathrm{CN}^{1 / 4}
$$

where $\mathrm{C}$ is a positive absolute constant.

Finally, a quantitative form of Ramsey's theorem says:

Theorem 1.3*. Let $|V|=n, S=[V]^{<}, A_{k}=\left\{A: A=[X]^{2}, x S\right.$, $|X|=k\}, G=\{g: S \rightarrow\{+1,-1\}\}$. Define $\Delta_{g}(A)=\sum_{x \in A} g(x)$. Then

$$
\Delta_{G}(A)=\left(\begin{array}{l}
k \\
2
\end{array}\right) \text { if } k<\frac{\log n}{2 \log 2} .
$$


2 PARTITIONS OF THE SET OF INTEGERS AND LATTICE POINTS IN $\mathrm{R}^{\mathrm{n}}$ We start by discussing results for arithmetic progressions.

(a) For arithmetic progressions we have both Ramsey type and discrepancy theorems, both expressing in a certain sense that we can not have too good partitions of the integers. In the case of Ramsey-type theorems this means that for any partition we must have a monochromatic arithmetic progression. The discrepancy-theorems mean that for any partition there exists an arithmetic progression, where one class has a large preponderance. So it should be clear that the seemingly qualitative difference between these statements is actually a quantitative one. If we focus on the short arithmetic progressions, the situation is so bad, that we must have an arithmetic progression where all but one colors are missing. If we focus on longer arithmetic progressions, this changes to a weaker preponderance phenomenon. This viewpoint helps to realize that we still have gaps in our knowledge. We will formulate the problems explicitly later.

(b) The methods used in the proofs of these results are good illustrative examples of the fascinating and fruitful relationship between the different fields. We will not give proofs, we just mention that e.g. in the discrepancy theorem 2.3 (below) for the lower bound Roth (1964) used a deep analytic method. Recently, an ingenious argument using only a combinatorial hypergraph theorem has been given by Beck (1981 a) which shows that Roth's lower bound is nearly sharp.

(c) In Ramsey theory van der Waerden's theorem for arithmetic progressions is widely generalized in different ways. However this is not the case with the discrepancy theorems. Having this common setting of the results on arithmetic progressions, we may again realize the gap, for quite a few general structures we know Ramsey-type generalizations but no discrepancy theorems.

Now we list a few theorems which may justify what we said above. Of course, here we can give just a sample of the results. First we mention the celebrated theorem of van der Waerden (1927):

Theorem 2.1. (van der waerden). If $\mathrm{N}>\mathrm{W}(\mathrm{k}, \mathrm{r})$, then for every $r$-coloming of $[N]$ at least one class must contain an arithmetic progression of length $k$.

Though van der Waerden's theorem appears to be a Ramsey-type theorem, actually there is another reason for its validity. Erdos and 
Turán conjectured already in 1936, that a density theorem is in the background. More exactly, they conjectured the following.

Let $r_{k}(n)$ denote the greatest integer $r$ such that there exists a sequence

$$
1 \leqq a_{1}<a_{2}<\ldots<a_{r} \leqq n
$$

which does not contain an arithmetic progression of length $k$. Then $r_{k}(n)=0(n)$.

This was proved by Roth (1952) for $k=3$ and it was a great achievement when Szemerédi (1973) proved it for arbitrary $k$.

Theorem 2.2. (Szemerédi). For every $k \geqq 3$

$$
r_{k}(n)=o(n)
$$

Remark. No good estimates for $W(k, r)$ resp. for $r_{k}(n)$ are known. A lower bound is

$$
W(k, 2)>\frac{2^{k}}{k} .
$$

However, the best known upper bound grows faster than

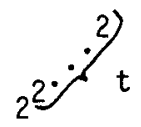

for any $t$. For reference see ErdOs \& Graham (1980).

Roth (1964) started to study the discrepancy problems for arithmetic progressions.

Let $P$ denote the set of arithmetic progressions in $[\mathrm{N}]$ and $g:[N] \rightarrow\{+1,-1\}$ a two-coloring of $[N]$. Let $P \in P$. Set

$$
\begin{aligned}
& \Delta_{g}(P)=\sum_{x \in P} g(x), \\
& \Delta_{g}(P)=\max _{P \in P}\left|\Delta_{g}(P)\right|
\end{aligned}
$$

and 


$$
\Delta(P ; N)=\min _{g}\left|\Delta_{g}(P ; N)\right|
$$

In the next theorems $c$ is always a positive absolute constant.

Theorem 2.3. (Roth (1964)). For any two-coloring

g : $[N] \rightarrow\{+1,-1\}$

$$
\Delta_{g}(P)>\mathrm{CN}^{1 / 4}
$$

Roth conjectured that for every $g:[N] \rightarrow\{+1,-1\} \quad \Delta_{g}(P)>$ $>c_{\sqrt{N}}$. This was disproved by Sárközy (1972). Recently Beck (1981 c) proved by an ingenious combinatorial argument that Roth's lower bound is nearly sharp. (See the combinatorial lemma in $\S 4$. )

Theorem 2.4. (Beck).

$$
\Delta(P ; N)<C N^{1 / 4}(\log N)^{5 / 2}
$$

There are different variations of the discrepancy theorems for arithmetic progressions. Actually Roth proved a more general theorem, which shows that the discrepancy of arithmetic progressions is large on average. A quantitative form of this is given in the following

Theorem 2.5. (Roth (1964)). Let

$$
P_{h, q}(m)=\{1 \leqq a \leqq m ; a \equiv h(\bmod q)\}
$$

Let $A \subseteq[N]$ be fixed and let

$$
\Delta_{h, q}(m ; A)=\Delta_{h, q}(m)=\mid\left(A \cap P_{h, q}(m)\left|-\frac{A}{|N|} P_{h, q}(m)\right| \mid .\right.
$$

Set

$$
v_{q}(m)=\sum_{k=1}^{q} \Delta_{h, q}^{2}(m)
$$

Then for any integer Q

$$
\sum_{q=1}^{Q} q^{-1} \sum_{m=1}^{N} v_{q}(m)+Q \sum_{q=1}^{Q} v_{q}(N) \gg \frac{A}{T_{N}}\left(1-\frac{A}{T_{N}}\right) Q^{2} N
$$


Quoting Roth, this theorem says that a sequence $A$ cannot be well distributed simultaneously among and within all congruence classes. (The choice $Q=N^{1 / 2}$ yields Theorem 2.3.)

In Roth's theorem the lower bound for the discrepancy depends on the ratio of the partition. But we do not have any information about the difference $d$ of the arithmetic progressions of large discrepancy. llow we list a few theorems concerning this problem.

Theorem 2.6. (Roth (1964)). Given any $\mathrm{S}: N \rightarrow\{+1,-1\}$ for every integer $k$ there is an arithmetic progression $P$ of difference $\mathrm{d}>\mathrm{k}$ such that

$$
\Delta_{g}(P)>c \sqrt{d}
$$

Beck \& Spencer (1983), using purely combinatorial arguments, proved that Roth's lower bound is nearly sharp. One of their results which gives upper bound for $\Delta(P)$ depending on the difference $d$ is the following.

Theorem 2.7. (Beck \& Spencer (1983)). Let $\varepsilon>0$ be arbitrary. Given any $\mathrm{n}>\mathrm{n}_{0}(\varepsilon)$ there is a two-coloring $\mathrm{g}: N \rightarrow\{+1,-1\}$ such that for any arithmetic progression $P$ of difference $n^{\varepsilon}<d<n$ and of arbitrary zength

$$
\Delta_{g}(P)<c \sqrt{d}(\log n)^{7 / 2}
$$

Probzem. Let $P_{t}$ denote the set of arithmetic progressions of length $t$. By Roth's theorem we know that given any two-coloring $\mathrm{g}:[\mathrm{N}] \rightarrow\{+1,-1\}$, there must be an arithmetic progression with discrepancy $\Delta_{\mathrm{g}}(P)>\mathrm{CN}^{1 / 4}$ and hence of length $t>\mathrm{CN}^{1 / 4}$. Probzem. What happens if $c \log N<t<N^{\varepsilon}$ ? Find upper and lower bounds for $\Delta\left(P_{t}\right)$.

The first generalization of van der Waerden theorem was given by Gallai (1931). Let $A=\left\{v_{1}, \ldots, v_{k}\right\}$ be a subset of $R^{m} . B=\left\{x_{1}, \ldots, x_{k}\right\}$ is homothetic to $A$ if, under a suitable ordering of $B$, there exist $\lambda \in R, \lambda \neq 0$ and an $a \in R^{m}$ so that

$$
x_{i}=\lambda v_{i}+a, \quad 1 \leqq i \leqq k
$$


Theorem. (Galzai). Let $A$ be an arbitrary finite subset of $R^{m}$. Given any $r$-coloring of $R^{m}$ there exists a monochromatic $B \subset R^{m}$ homothetic to A.

A further generalization is the Hales \& Jewett (1963) theorem, which is considered as one of the most basic theorems in Ramsey-theory. (See Graham \& al. (1980).)

Definition. Let

$$
c_{t}^{n}=\left\{\left(x_{1}, \ldots, x_{n}\right) ; x_{i} \in\{0, \ldots, t-1\}\right\} .
$$

A line in $c_{t}^{n}$ is a set of suitably ordered points $x_{0}, \ldots, x_{t-1}, x_{i}=$ $=\left(x_{i 1}, \ldots, x_{i n}\right)$ so that for each coordinate $j, 1 \leqq j \leqq n$ either

$$
x_{o j}=x_{1 j}=\ldots=x_{t-1, j}
$$

or

$$
x_{\ell j}=\ell \quad \text { for } 0 \leqq \ell<t
$$

and the latter holds for at least one $j$.

Theorem. (Hales \& Jewett (1963)). For every $r, t$ there exists a least integer $\mathrm{HJ}(r, t)$ so that, for $\mathrm{N}>\mathrm{HJ}(r, t)$ if the vertices of $c_{t}^{N}$ are $r$-colored, then there exists a monochromatic line.

This is a corollary of a much more general Ramsey theorem (Graham-Leeb-Rothschild (1972) which also implies the following theorem.

Let $A$ be an arbitrary finite field and let $A^{n}$ be the $n$ dimensional space over $A$. For every $r, t, k$ positive integers there exists an $N_{t}(k ; r)$ so that if the $t$-dimensional linear subspaces of $A^{r}$ are $r$-colored then there exists a $k$-dimensional vector space all of whose $t$-dimensional linear subspaces have the same color.

Remark. It is easy to see that van der Waerden's theorem is also a corollary of the Hales-Jewett theorem. For this consider the integers $\mathrm{a}, 0 \leqq \mathrm{a}<\mathrm{t}^{\mathrm{N}}$ and the base- $\mathrm{t}$-representation.

$$
a=\sum_{i=0}^{N} a_{i} t^{i}, \quad 0 \leqq a_{i}<t .
$$


An $r$-coloring of $\left[t^{N}\right]$ induces an $r$-coloring of $c_{t}^{N}$. This has a monochromatic line, if $N$ is large enough. But it is easy to see that a monochromatic line corresponds to a monochromatic arithmetic progression of length $t$.

Problem. We have the discrepancy-results for arithmetic progressions. Is it possible to get discrepancy-results for $C_{t}^{n}$ ?

A common generalization of yan der Waerden's and Schur's theorem was given by R. Rado (1933 a,b).

Let $C$ be an $m \times n$ matrix of integer entries, $C \underline{x}=0 \quad a$ system of homogeneous linear equations in the variables $x_{1}, \ldots, x_{n}$. We say that $C$ is regular if given any finite coloring $N$, there exists $\underline{x}=\left(x_{1}, \ldots, x_{n}\right)$ such that $\underline{C} \underline{x}=0$ and $x_{1}, \ldots, x_{n}$ are the same color.

We say that the matrix $C$ satisfies the column-condition if after a suitable rearranging of the column-vectors $c_{1}, \ldots, c_{n}$ one can find $1 \leqq k_{1}<\ldots<k_{t}=n$ such that for

$$
A_{i}=\sum_{j=k_{j-1}+1}^{k_{i}} c_{j}
$$

we have $A_{1}=0$ and for $1<i \leqq t, A_{i}$ is a linear combination of $\underline{c}_{1}, \ldots$ $\ldots, c_{k_{j}-1}$.

Theorem. (Rado). C is regular on $N$ iff $\mathrm{C}$ satisfies the column-condition.

Remark. It is easy to see that Schur's theorem is a consequence of Rado's theorem. Namely, if we have a single equation

$$
a_{1} x_{1}+\ldots+a_{n} x_{n}=0
$$

the column-condition means that some nonempty subset of the $c_{\boldsymbol{i}}$ sums to zero. Evidently $x+y-z=0$ satisfies this condition.

To obtain van der Waerden's theorem as a special case we consider the system

$$
\begin{aligned}
& x_{2}=x_{1}+d \\
& \vdots \\
& x_{k}=x_{k-1}+d .
\end{aligned}
$$


Similarly as in the case of the Hales \& Jewett theorem Rado's theorem is not accompanied by general discrepancy theorems.

\section{PARTITIONS ON GRAPHS}

The results here belong partly to Ramsey theory, partly to extremal graph theory. As there are excellent monographs on both subjects (Graham, Rothschild \& Spencer (1980), Bollobás (1980)), we will mention only those basic results which are relevant for our present aim. However we will give a more detailed discussion of results which are more recent ones and are not contained in the books mentioned above.

First we consider partitions of $[n]^{l}$, with respect to the family of complete graphs.

Most of the results refer to the case $\ell=2$. Still, we formulate Ramsey's theorem for arbitrary $\ell$.

Theorem 3.1. (Ramsey (1930)). For alz integers $\ell, r$, $\mathrm{k}_{1}, \ldots, \mathrm{k}_{\mathrm{r}}$ there exists a minimal integer $\mathrm{R}_{\ell}\left(\mathrm{k}_{1}, \ldots, \mathrm{k}_{r}\right)$ with the following property:

$$
\text { if } n>R_{\ell}\left(k_{1}, \ldots, k_{r}\right) \text {, given any } r \text {-coloring } g:[n]^{l} \rightarrow
$$
$\rightarrow\{1, \ldots, r\}$, there exist on $i, 1 \leqq i \leqq r$ and a set $S \subset[n]$ such that $|S|=k_{i}$ and $[S]^{l}$ is colored $i$.

Little is known about the Ramsey-numbers $R_{\ell}\left(k_{1}, \ldots, k_{\ell}\right)$. Here we mention just two results for the simplest cases, which are relevant in our discussion:

$$
2^{k / 2}<R_{2}(k, k)<4^{k}
$$

$$
c k^{2}(\log k)^{-2}<R_{2}(3, k)<c k^{2}(\log k)^{-1}
$$

(see Graham, Rothschild \& Spencer (1980)).

There are several interpretations of Ramsey's theorem. Graham, Rothschild \& Spencer (1980) give a deep analysis of this question. We repeat only their quotation of Burkill \& Mirsky (1973). "There are numerous theorems in mathematics which assert, roughly speaking, that every system of certain class possesses a large subsystem with a higher degree of organization than the original system." We emphasize here another aspect of Ramsey's theorem. 
Suppose we want to two-color the edges of $k_{n}$ by red and blue so that in every $k_{k}$ we have about the same number of red and blue edges. Now the situation is similar to the case of arithmetic progressions. By $\left(^{*}\right)$ we know that if $k<\frac{1}{2 \log 2} \log n$, the situation is so bad that for any two-coloring we must have a monochromatic $k_{k}$. As $k$ gets larger, we can two-color more uniformly, with respect to the $k_{k}$ 's, though we still have the preponderance phenomenon.

A quantitative form of this is given by the following theorem. Theorem 3.2. (Erdös-Spencer (1972)). Let $\mathrm{g}:[n]^{2} \rightarrow\{+1,-1\}$

and

$$
\Delta_{g}(n ; t)=\max _{S \subset[n]}\left|\sum \underset{g}{|S| \leqq t}\right| x \in\left[S j^{2} \mid\right.
$$

Define

$$
\Delta(n ; t)=\min _{g} \Delta_{g}(n ; t)
$$

Then

$$
\Delta(n ; t)=\left(\begin{array}{l}
t \\
2
\end{array}\right), \text { if } t \leqq \frac{\log n}{2 \log 2}
$$

and

$$
10^{-3} t^{3 / 2} \sqrt{\log \frac{5 n}{t}} \leqq \Delta(n ; t) \leq t^{3 / 2} \sqrt{\log \frac{5 n}{t}} \text {, if } t>\frac{2 \log n}{\log 2} \text {. }
$$

Corozzary. Let

$$
\Delta(n)=\min _{g} \max _{S \subset[n]}\left|\sum_{x \in[S]^{2}} g(x)\right|
$$

Then

$$
c_{1} n^{3 / 2}<\Delta(n)<c_{2} n^{3 / 2}
$$

where $c_{1}, c_{2}$ are positive absolute constants.

This theorem has a generalization for hypergraphs. 
Theorem 3.4. (Erdos \& Spencer). Let $g:[n]^{k} \rightarrow\{+1,-1\}$. Set

$$
\Delta(n ; t)=\min _{g} \max _{\substack{S \subset[n] \\|S| \leq t}}\left|\sum_{x \in[S]} g(x)\right|
$$

Then

$$
c_{1}\left(\begin{array}{l}
t \\
k
\end{array}\right) \leqq \Delta(n ; t) \leqq\left(\begin{array}{l}
t \\
k
\end{array}\right) \text { if } t \leqq(\log n)^{\frac{1}{k-1}}
$$

and

$$
c_{2} t^{\frac{k+1}{2}} \sqrt{\log \frac{5 n}{t}} \leqq \Delta(n ; t) \leqq t^{\frac{k+1}{2}} \sqrt{\log \frac{5 n}{t}}
$$

where $\mathrm{c}_{1}, \mathrm{c}_{2}$ are positive absolute constants.

The case $k_{1}=\ldots=k_{\ell}$ of Ramsey theorem is called "symmetric case", otherwise it is called "asymmetric".

We know that - concerning the theorems above - the best colorings are the random ones. Here "best" means that the largest monochromatic complete graph is as small as possible, resp. the discrepancy in the $K_{t}$ 's is as small as possible. In the symmetric case if $r=2$ this means that in the best colorings each color class contains about the half of the edges.

Hence if we consider partitions of given ratio, $\alpha, 1-\alpha$ and $\alpha \frac{1}{2}$, the same discrepancy phenomenon will appear, but the quantitative results will be different.

As to the asymmetric case, here we suppose $k=0(\ell)$ and $n \leqq R(k, l)-1$. Let us consider the two-colorings of $k_{n}$ which contain neither a red $k_{k}$ nor a blue $k_{\ell}$. It is easy to see that if $\ell$ is much larger than $k$, then the number of red edges in such a coloring will be much smaller than the number of blue edges. The ratio tends to 0 as $\frac{k}{l} \rightarrow 0$. The conjecture is that for fixed $k, R(k, l) \approx \ell^{k-1+0(1)}$.

All these facts make plausible that if we have a restriction on the number of edges in the graph (on the ratio of the partition) this will increase the size of the complete graphs we can ensure.

Another aspect of the results is the following:

If we make a comparison between van der Waerden's theorem and Roth's theorem for arithmetic progressions on the one hand and Ramsey's 
theorem, Erdys \& Spencer theorem for complete graphs on the other hand, the similarity is clear.

In both cases we have a set $S$ and a family $A \subseteq 2^{S}$. For any two-coloring of $S$ among the small sets in $A$ there must be a monochromatic one, and among the larger sets still there must be one in which there is a certain discrepancy.

However, the background of the results for the two structures, at least for the monochromatic case is different.

For arithmetic progressions actually a density theorem yields the resuit. Namely Szemerédi's theorem means that for every $c>0$, if $n>n_{0}(k), A \subseteq[n]$, and $|A|>c n$, then $A$ must contain an arithmetic progression of length $k$. Since for every two-coloring at least one color-class contains more than $\frac{n}{2}$ elements, we must have an arithmetic progression of length $k$ in it.

Although we have a density theorem for complete graphs, (Turăn's theorem) we need more than half of the edges to ensure the existence of a complete $k_{k}$. Therefore, if we consider the two-colorings of the edges of $K_{n}$, just a density argument will not be enough to ensure the existence of a monochromatic $k_{k}$. This is the reason why we have a new class of problems and results for graphs.

Let $H^{k}$ be an $\ell$-uniform hypergraph. Let $f\left(n ; H^{k}\right)$ be the minimal integer $e$ such that every $k$-uniform hypergraph on $n$ vertices and more than e edges contains a subgraph isomorphic to $H^{k}$. A $G_{n}^{k}(V ; E)$ is called an extremal graph belonging to $H^{k}$, if $|V|=n$, $|E|=f\left(n ; H^{k}\right)$ and $G_{n}^{k}$ does not contain sugraphs isomorphic to $H^{k}$.

The determination (or estimation) of $f\left(n ; H^{k}\right)$ is the fundamental problem of extremal graph theory started by Turán (1941).

As to the density theorems for graphs, we mention only results which are relevant here.

First we consider the case $\ell=2$ and $H^{2}=K_{k}$.

Theorem 3.5. (Turơn (1941)). Let $n \equiv r \bmod (k-1), 0 \leqq r<k-1$.

Then

$$
f\left(n ; k_{k}\right)=\frac{1}{2} \frac{k-2}{k-1}\left(n^{2}-r^{2}\right)+\left(\begin{array}{l}
r \\
2
\end{array}\right)
$$

There is a unique extremal graph, the complete $(\mathrm{k}-1)$-partite graph having $\left[\frac{n}{\mathrm{k}-1}\right]$ resp. $\left[\frac{\mathrm{n}}{\mathrm{k}-1}\right]+1$ vertices in each class. 


\section{Observe that}

(1) as we said above, even in the case $k=3$ we need more than half of the edges to ensure the existence of a $k_{k}$.

(2) From our point of view it is important that the extremal graph contains a very large independent set of size $\left[\frac{n}{k-1}\right]$.

These indicate, too, that if we have a restriction on the number of edges in one color-class (on the ratio of the partition), then for the asymmetric case this will change the size of the complete graphs we can ensure in one color-class.

To formulate a slightly more general problem consider partitions of $[n]^{2}$ of ratio $\alpha, 1-\alpha$. For which pairs $(k, l)$ is it true, that either the first class contains a $k_{k}$ or the second class contains a $K_{\ell}$. (Evident]y, by Ramsey theorem, with $n \rightarrow \infty \max (k, \ell) \rightarrow \infty$.)

Or more generally, let $R T(n ; k, \ell)$ be the set of integers $e$, for which there is a coloring of $[n]^{2}$ such that the number of red edges is $e$ and neither a red $k_{k}$ nor a blue $k_{\ell}$ exists. What can be said about $R T(n ; k, l)$ ? In particular, we are interested in the value of

$\max R T(n ; k, l)$ and $\min R T(n ; k, l)$.

Results which give information on this question are called Ramsey-Turán type theorems.

Since we do not have too much knowledge about the Ramsey numbers $R(k, l)$ one can expect that the most we can have are asymptotic results.

We start with a result of Erdös and Szemerédi on the symmetric case.

Theorem 3.6. (Erabis \& Szemerédi (1972)). Let $r \geqq 2$. Let $G(V ; E)$ be a graph with $|V|=n,|E| \leqq \frac{1}{r}\left(\begin{array}{l}n \\ 2\end{array}\right)$. There exists a positive $a b-$ solute constant $\mathrm{C}$ so that either $G$ or its complement contains a $\mathrm{K}_{\mathrm{k}}$ with $k>c \frac{r}{\log r} \log n$.

Remark. In the present setting the above theorem can be

formulated as follows. Let

$$
G^{*}=\left\{g \mid g:[n]^{2} \rightarrow\{+1,-1\}, \sum_{x \in[n]} g(x)=\left(1-\frac{2}{r}\right)\left(\begin{array}{l}
n \\
2
\end{array}\right)\right\} .
$$


Set

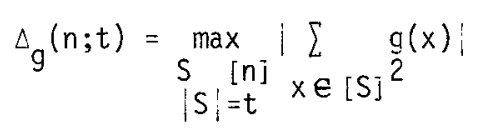

and

$$
\Delta^{(r)}(n ; t)=\min _{g \in G^{\star}} \Delta_{g}(n ; t) .
$$

Then

$$
\Delta^{(r)}(n ; t)=\left(\begin{array}{l}
t \\
2
\end{array}\right) \text { if } t<c \frac{r}{\log r} \log n
$$

Problem. Find a commion generalization of Theorem 4.2 and Theorem 4.3: give estimates for $\Delta^{r}(n ; t)$ if $t>c \frac{r}{\log r} \log n$.

Remark 2. The Erdys-Szemerédi theorem gives a partial answer to the following question:

Let $f(n ; r)$ be the largest integer $k$ such that for any $r$ coloring of $[n]^{2}$ there exists an $S \subset[n],|S|=k$ for which $[S]^{2}$ meets at most $r-1$ color-classes. Since at least one color-class contains not more than $\frac{1}{r}\left(\begin{array}{l}n \\ 2\end{array}\right)$ edges, by the Erdos-Spencer theorem $f(n ; r)>$ $>c \frac{r}{\log r} \log n$.

Problem. Let $1 \leqq s<r$. Let $f(n ; r, s)$ denote the largest integer $k$ such that for any $r$-coloring of $[n]^{2}$ there exists an $S \subset[n],|S|=k$, for which $[S]^{2}$ meets at most $s$ color-classes. Find upper and lower bounds for $f(n ; r, s)$.

Let

$$
R T(n ; k, l)=\max R T(n ; k, l) .
$$

As to the function $\operatorname{RT}(n ; k, \ell)$ in the asymmetric case, most of the results are asymptotic estimates for the case when $\ell$ is replaced by a function of $n$ which is $o(n)$. For this we will use the symbol $\mathrm{RT}(n ; k, o(n))$.

For $t \geqq 3$ put 


$$
\begin{array}{ll}
a_{t}=\frac{1}{2} \frac{t-3}{t-1}=\frac{1}{2} \frac{3 t-9}{3 t-3} & \text { if } t \text { is odd } \\
a_{t}=\frac{1}{2} \frac{3 t-10}{3 t-4} & \text { if } t \text { is even. }
\end{array}
$$

(The sequence $0=a_{3}, a_{4}, \ldots=0, \frac{1}{8}, \frac{1}{4}, \frac{2}{7}, \ldots$ is strictly increasing.)

Theorem 3.7. (Erd8s-HajnaL-T.Sos-Szemerédi (1983)). For $t \geqq 3$

$$
R T(n ; t, o(n))=a_{t} n^{2}(1 ; 0(1))
$$

The investigation of $\mathrm{RT}(n ; t, o(n))$ started in T. Sós (1969) and Erdds - T. Sós (1969). The above result for $t$ odd was proved in Erdüs \& T. Sós (1969). The case when $t$ is even turned out to be much harder. It w:as proved only much later in Szemerédi (1973) and in Bollobás \& ErdDs (1976) that $R T(n ; 4, o(n))=\frac{n^{2}}{8}(1+o(1))$. (Szemerédi (1973) gives the upper bound and Bollobăs \& Erdös (1976) the construction yielding the lower bound.

Results for an other range of the parameters $k, \ell$, or in other words, for min $R T(n ; k, o(n))$ are given in Ajtai-Komlós-Szemerêdi (1981), Komlós-Pintz-Szemerédi (1982), Ajtai-Erdðys-Komlós-Szemerēdi (1981), Ajtai-Komiós-Pintz-Spencer-Szemerēdi (1983).

Theorem 3.8. (Ajtai-KomZós-Szemerédi (1981)). Let $G(V ; E)$ be a graph of $n$ vertices, $\mathrm{e}$ edges. Let $\mathrm{t}$ denote the average degree: $\mathrm{t}=\frac{2 \mathrm{e}}{\mathrm{n}}$ and $\alpha(G)$ denote the maximum size of an independent set of vertices (independence or stabizity number).

If $G$ does not contain a triangle, then

$$
\alpha(t)>c \frac{n}{t} \log t
$$

(where $\mathrm{c}$ is a positive absolute constant).

The result is best possible as to the order of magnitude.

Remark. Without the assumption that $G$ is triangle-free, by a simply greedy-algorithm argument only $\alpha(t)>c \frac{n}{t}$ would follow. 
Theorem 3.9. (KomZós-Pintz-Szemerédi (1982)). Let $H^{3}(\mathrm{~V} ; \mathrm{E})$ be a 3 -uniform hypergraph of $\mathrm{n}$ vertices and $\mathrm{e}$ edges. Let $\mathrm{t}$ denote the average degree; $\mathrm{t}=\frac{3 \mathrm{e}}{\mathrm{n}}$ and let $\alpha(G)$ denote the maximum size of an independent set.

If $H^{3}(V ; E)$ does not contain cycles of length $\leqq 4$, (in $\alpha$ precise meaning, which we do not give here)

$$
\alpha(G)>c \frac{n}{\sqrt{t}} \log t
$$

(Here $\mathrm{c}$ is a positive absolute constant.)

See also ErdBs-Komlós-Pintz-Spencer-Szemerédi (1983).

Remark. It is worth mentioning that the Ramsey-Turán type theorems were considered because of different applications. There is a sequence of papers by Eräbs-Meir-T. Sós-Turăn (1971), (1972 a), (1972 b), where Turán's theorem is employed to obtain results for the distribution of distances in metric spaces. Ajtaiet al. (1981) applied their theorem in the investigation of Sidon-sequences. The result in Komlóset al. (1982) was the key lemma to disprove a more than 20 year-old conjecture of Heilbronn for the minimum area of triangles determined by $n$ points in the unit square.

Remark. Observe that $R T(n ; k, 0(n)) \approx c_{k} f(n ; k)$ with a $c_{k}<1$. Surprisingly enough the situation for $l$-uniform hypergraphs is different if $\ell \geqq 3$. In Erdßs \& T. Sós (1982) it is proved that

$$
R^{(l)}(n ; k, o(n)) \approx f^{(l)}(n ; k)
$$

where $R T^{(\ell)}(n ; k, o(n))$ and $f^{(l)}(n ; k)$ has a similar meaning for $l \geqq 3$ as $R T(n ; k, o(n))$ and $f(n ; k)$ for $\ell=2$.

Now let us consider the discrepancy in colorings of the edges of $K_{n}$ with respect to general graphs.

Let the graphs $G_{1}, \ldots, G_{r}$ be fixed. Evidently it follows from Ramsey theorem that for $n$ large enough, for every $r$-coloring of $[n]^{2}$ there exists $i, 1 \leqq i \leqq r$ so that a copy of $G_{i}$ occurs in the $i$ th color. The problem is to find the least integer $n$ for which 
this holds. Let $R\left(G_{1}, \ldots, G_{r}\right)$ denote this least integer. The investigation of $R\left(G_{1}, \ldots, G_{r}\right)$ was started in Gerencsér \& Gyárfăs (1967) where they considered the case $r: 2, G_{1}=P_{k}, G_{2}=P_{\ell}$ (the paths of length $k$ resp. $\ell$ ).

Though the problem is more general, for some special class of graphs it is easier to get good estimates or even to get the exact values of $R\left(G_{1}, \ldots, G_{r}\right)$ than for $R\left(k_{1}, \ldots, k_{r}\right)$.

The Turán type results for arbitrary graphs are also relevant here. For all graphs $H^{2}$ with chromatic number $X\left(H^{2}\right)>2$ the asymptotic value of $f\left(n ; H^{2}\right)$ is known.

Theorem 3. 10. (Erd8s-Simonovits (1966)). Let $x\left(H^{2}\right)=k$

$$
f\left(n ; H^{2}\right) \approx f(n ; k)=\frac{1}{2} \frac{k-2}{k-1} n^{2}+o\left(n^{2}\right) .
$$

Even more is true.

Theorem 3.11. (Erabs (1967), Simonovits (1968). If $\mathrm{k}>2$, the extremal graphs (having $\mathrm{f}\left(\mathrm{n} ; \mathrm{H}^{2}\right)$ edges without containing subgraphs isomorphic to $\mathrm{H}^{2}$, can be made isomorphic by adding to and deleting from $\mathrm{o}\left(\mathrm{n}^{2}\right)$ edges the Turón-groph (the complete $(\mathrm{k}-1)$-partite graph having $\left[\frac{n}{k-1}\right]$ or $\left[\frac{n}{k-1}\right]+1$ vertices each class).

Remark. If $\mathrm{k}=2$, the above theorem gives only that

$$
f\left(n ; H^{2}\right)=o\left(n^{2}\right) \text {. }
$$

For most bipartite graphs the exact value of $f\left(n ; H^{2}\right)$ or even asymptotic formula for it is not known, and to determine it is among the most difficult problems in extremal graph theory.

Roughly speaking, the maximum number of edges a graph may have without containing $H^{2}$ as a subgraph, asymptotically depends onty on the chromatic number of $\mathrm{H}^{2}$.

Why are the above results relevant in the problems for $\operatorname{RT}\left(G_{1}, G_{2}\right)$ ? If $x\left(G_{1}\right)=x\left(G_{2}\right)=2, f\left(n ; G_{j}\right)=o\left(n^{2}\right) \quad(i=1,2)$. Hence a density theorem ensures the existence of a monochromatic $G_{1}$ or $G_{2}$. This means that the situation in this case is similar to the case of arithmetic progressions.

Remark. Here we discussed the following type of problems: 
(1) Ramsey-type problems;

(2) Turăn-type problems;

(3) Ramsey-Turăn-type problems;

(4) discrepancy-problems.

In all the four cases we considered the complete graphs as subgraphs. In cases (1) - (2) there are many results for other graphs too. In (2) we know that the chromatic number of a graph is the most relevant parameter which determines the behavior of $f(n ; G)$.

In (1) most of the results are for graphs $G_{1}, G_{2}$ when $\min _{i} x\left(G_{j}\right)=2$ (see Graham-Rothschild-Spencer (1980), and in the general case we do not know which parameters of the graphs $G_{1}, G_{2}$ determine the Ramsey-function $R\left(G_{1}, G_{2}\right)$.

For (3) in Erdös-Hajnal-T. Sós-Szemerédi (1983) the function $\operatorname{RT}(n ; H, O(n))$ is defined as the maximal e for which there exists a graph $G$ with $n$ vertices and $e$ eages, such that $H \nsubseteq G$ and the stability number of $C$ is $o(n)$. Not even asymptotic results for $\operatorname{RT}(n ; H ; O(n))$ are known in the general case. The lower and upper bounds proved in Eräbs-Hajnal-T. Sós-Szemerécii show that here the arboricity number of the graph $H$ is relevant.

In (4) only the complete graphs were considered. It would be interesting to have discrepancy theorems also for other graphs.

\section{PARTITIONS IN $\mathrm{R}^{\mathrm{n}}$. CLASSICAL THEORY OF UNIFORMLY DISTRIBUTED} SEQUENCES

To begin with the history we have to go back to the seventeenth century when Huygens wanted to give a mechanical model for the solar system using a system of gears. Each gear represented a planet.

The number of teeth on the gears had to be chosen so that the ratio of these numbers were close to that of the periods of revolution of the represented planets. At the same time the number of teeth on each gear was limited. So the mathematical problem was the following: 
Given a real number $\alpha$ and $\mathbb{N}$, find integers $0 \leqq p, q \leqq N$ so that $\left|\alpha-\frac{p}{q}\right|$ is as small as possible.

This was one of the germs of the theory of diophantine approximation. The theory developed much later, mainly due to the works of ostrovski, Hecke, Hardy, Littlewood etc. It became clear that the approximability property of $\alpha$ depends on the partial quotients ( $a_{k}$ digits) of its continued fraction expansion $\alpha=\frac{1}{a_{1}+\frac{1}{a_{2}+\ldots}}$ (denoted by $\alpha=$ $\left.=\left[a_{1}, a_{2}, \ldots\right]\right)$. It became also clear that the approximability property of $\alpha$ - is closely related to the distribution of the sequence $(\{n \alpha\})$ in $(0,1)$. ( $\{n \alpha\}$ denotes the fractional part of $\alpha)$. Evidently, for every irrational $\alpha$, the sequence $\{n \alpha\}$ is everywhere dense in $(0,1)$. The fact that it is uniformly distributed, expresses a stronger property. Let $E^{k}$ denote the $k$-dimensional unit cube $[0,1]^{k}$. $I=X_{i=i}^{k}\left[a_{i}, b_{i}\right]$ be a box in $E^{k}, I(\underline{x})={\underset{j=1}{X}\left[0, x_{i}\right],|I|}_{i}$ be the Lebesgue measure of $I$.

Let $\omega=\left(u_{n}\right)$ be a sequence in $E^{k}$. We write $Z_{\omega}(N ; I)$ (or simply $Z(N ; I)$ ) for the number of $u_{i} \in I, 1 \leqq i \leqq N$. $E^{k} \quad$ if for every box $I \subset E^{k}$

$$
\lim _{N \rightarrow \infty} \frac{Z_{\omega}(N ; I)}{N}=|I|
$$

holds.

An equivalent definition is the following.

Definition 2. Let $R\left(E^{k}\right)$ denote the set of Riemann-integrable functions on $E^{k}$. The sequence $\left(u_{n}\right)$ is uniformly distributed in $E^{k}$ if for every $f \in R\left(E^{k}\right)$

$$
\lim _{N \rightarrow \infty} \frac{1}{N} \sum_{n=1}^{N} f\left(u_{n}\right)=\int_{E^{k}} f(x) d x
$$

The second definition seems to be less natural, however it is a more fruitful one. It indicates why uniformly distributed sequences are important in the theory of numerical integration (see Remark 4.1). 
Further observe that we obtain an equivalent definition if we assume that (4.2) holds for a dense subset of $R\left(E^{k}\right)$. This indicates how the concept of uniformly distributed sequences can be generalized to topological groups.

For the general theory of uniformly distributed sequences see the excellent book of Kuipers-Niederreiter (1974).

Put $\quad \Delta_{N}^{\omega}(I)=|Z(N ; I)-N| I||$

and

$$
\begin{aligned}
& \Delta_{N}^{w}=\left\|\Delta_{N}^{\omega}\right\|_{\infty}=\sup _{I \subset E^{k}} \Delta_{N}^{w}(I) ; \\
& \left.\left\|\Delta_{N}\right\|_{p}=\left.\left(\int_{E^{k}} \Delta_{N}^{\omega}(I)(x)\right)\right|^{p} d x\right)^{\frac{1}{p}} .
\end{aligned}
$$

$\Delta_{N}$ resp i| $\Delta_{N} j p$ measures (in different norms) the discrepancy of the sequence $u_{1}, \ldots, u_{N}$, their behavior for $N \rightarrow \infty$ measures the irregularity of the distribution of the infinite sequence $\left(u_{n}\right)$.

In the quantitative theory of uniform distribution a central problem is the investigation of the order of magnitude of the discrepancyfunctions $\quad \mid \Delta_{N} \|_{p}, \Delta_{N}$.

It is easy to see that a sequence $\omega$ is uniformly distributed in $E^{k}$ iff $\Delta_{n}^{\omega}=0(N)$. But how small can $o(N)$ be?

The quantitative theory of uniformiy distributed sequences started with the following conjecture of van der Corput (1935 a).

For an arbitrary sequence in $[0,1)$, sup $\Delta_{N}=\infty$. This means that no sequence can be "too evenly" distributed. This was proved by van Aardenne Ehrenfest (1945) who showed that for an arbitrary sequence $\left(u_{n}\right)$ for infinitely many $\mathrm{N}$

$$
\Delta_{N}>c(\log \log N)(\log \log \log N)^{-1}
$$

Roth (1954) strengthened this result. He proved the following more general theorem. Theorem 4.1. (Roth (1954)). A. For an arbitrary infinite se-
quence $\left(u_{n}\right)$ in $\mathrm{E}^{\mathrm{k}}$ and for every $\mathrm{N}>\mathrm{N}_{0}$ 


$$
\max _{1 \leq n \leq N}\left\|\Delta_{n}\right\|_{2}>c_{k}(\log N)^{\frac{k}{2}} .
$$

B. For $\mathrm{N}$ arbitrary points $\mathrm{u}_{1}, \ldots, \mathrm{u}_{\mathrm{N}}$ in $\mathrm{E}^{\mathrm{k}}$

$$
\| \Delta_{N} i_{2}>c_{k}^{\prime} \log _{N}^{\frac{k-1}{2}} \text { if } N>N_{0} \text {. }
$$

(Here $c_{k}, c_{k}^{\prime}$ are positive absolute constants.)

For $k=2$ Davenport (1956) and for $k \geqq 3$ Roth (1979), (1980) proved that (apart from a multiplicative constant) these results on $\mid A_{\mathrm{N}} \|_{2}$ are sharp.

The theorem implies (in a precise quantitative form), that the irregularity of the distribution increases with the dimension.

The problem of finding bounds for the discrepancy in supremum norm is more difficult. Since $\Delta_{N} \geqq \Delta_{N} l_{2}$, the preceding results give some lower bounds on $\Delta_{N}$. For infinite sequences sharp results are known only for $k=1$, for finite sequences for $k=2$.

Theorem 4.2. (Schmidt (1972)). A. For arbitrary infinite sequence $\left(u_{n}\right)$ in $(0,1)$ and for every $N>N_{0}$

$$
\max _{1 \leq n \leq N} \Delta_{n}>c \log N
$$

B. For arbitrary $N$ points $u_{1}, \ldots, u_{N}$ in $E^{2}$

$$
\Delta_{N !}>C^{\prime} \log N
$$

where $c, c^{\prime}$ are positive absolute constants.

This result is best possible apart from the multiplicative constant. E.g. if $u_{n}=\{n \alpha\}$ where $\alpha$ is an irrational number of bounded partial quotients $\left(a_{k} \leqq K, k=1,2, \ldots\right)$, then for every $N, \Delta_{N}<c_{K} \log N$. Similarly, for the $N$ points $u_{n}=\left\{\left\{n \alpha, \frac{n}{N}\right\}, 1 \leqq n \leqq N\right.$ in $E^{2} \Delta_{N}<c_{K} \log N$.

We have mentioned that uniformly distributed sequences play an important role in the theory of numerical integration. The first result on this is the following.

Theorem. (Koksma inequality (1942/43)). Let $f$ be a function 
on $[0,1)$ of bounded variation $v(f)$ and $u_{1}, \ldots, u_{N}$ be $N$ given points in $[0,1)$ with discrepancy $\Delta_{\text {f. }}$. Then

$$
\left|\frac{1}{N} \sum_{n=1}^{N} f\left(u_{n}\right)-\int_{0}^{1} f(t) d t\right| \leq V(f) D_{N} \frac{1}{N}
$$

Koksma's inequality has various generalizations, to higher dimensiorstoo. These theorems show that in computing integrals (especially in higher dimensions) well distributed sequences are of great importance. The smaller the discrepancy is, the better the approximation will be. This led to the criticism of Monte Carlo methods, where randomly generated sequences are used. Since a random sequence has discrepancy $\sqrt{\mathrm{N} \log \log N}$, it is more advantageous to work with we11-distributed deterministic sequences of discrepancy of a power of $\log N$ (Niederreiter (1978)).

Remark. By an observation of Roth part $A$ and part $B$ of Theorem 4.1 and similarly of Theorem 4.2 are equivalent. Roth's argument (for $k=1$ ) is the following.

(a) Let $u_{1}, \ldots, u_{N}$ be a sequence of points in $[0,1)$. Consider a corresponding set $\left(u_{i}, \frac{i-1}{N}\right), 1 \leq i \leq N$ of $N$ points in $E^{2}$.

(b) Let $\left(x_{i}, y_{i}\right), 1 \leqq i \leqq N$ be a sequence of points in $E^{2}$, arranged so that $y_{1} \leqq y_{2} \leqq \ldots \leqq y_{N}$. Take the corresponding sequence $x_{1}, \ldots$ $\ldots, x_{N}$ in $[0,1)$.

In both cases the discrepancies of the two sequences are the same up to a universal constant factor.

Theorem 4.2. can be formulated as an assertion on partitions of a special finite hypergraph. (See Theorem 1.1*.) We obtain this finite version, if instead of $E^{2}$ (as underlying set) we take the $N \times N$ lattice. The continuous or discrete formulation makes no difference as long as the members of the family $A$ are the set of points in aligned rectangles. However, if we consider other families too, the situation changes. First$1 y$, it may depend on the underlying set, which families are worth considering. Secondly, it depends on the family which form fits better to the distribution problem or to the proof.

\section{GEOMETRICAL STRUCTURES}

In this section we discuss a variety of questions where the underlying set $S$ is either the $k$-dimensional unit cube $E^{k}$, or (in 
the discrete version) the $\mathrm{N} \times \mathrm{N}(\mathrm{N} \times \mathrm{N} \times \ldots \times \mathrm{N})$ lattice defined as the set of points with integer coordinates $1 \leqq k \leqq N, 1 \leq \ell \leq N$. A is a family of simple geometrical objects, as aligned or tilted rectangles, triangles, balls, etc. (see Erdys (1964)).

The distribution (or partition) problems will be formulated in the following two forms.

(a) Given $N$ points in $S$, how evenly can they be distributed with respect to the sets in $A$ ?

(b) Given $N$ points in $S$, how "good" can a two-coloring of these points be with respect to the sets in $A$ ?

All the theorems here give quantitative results on the weaker preponderance phenomenon: none of them is Ramsey-type (ensuring monochromatic subsets). Of course, one could consider the Ramsey-type results for lattice points mentioned in $\S 2$ as belonging to this group of problems, too (e.g. Roth, Hales \& Jewett, Szemerédi's theorem, etc.).

Let $U_{n}=\left\{u_{1}, \ldots, u_{N}\right\} \subset S=E^{2}, A$ be a family of subsets in $R^{k}$. Let $\mathbb{Z}\left(A ; U_{N}\right)$ denote the number of points $u_{i}, 1 \leq i \leq N$ in $A \in A$. Set

$$
\begin{aligned}
& \Delta\left(A ; U_{N}\right)=\left|\mathbb{Z}\left(A ; U_{N}\right)-N_{\mu}(A \cap S)\right| \\
& \Delta\left(A ; U_{N}\right)=\sup _{A \in A} \Delta\left(A ; U_{N}\right) \\
& \Delta_{N}(A)=\inf _{U_{N}} \Delta\left(A ; U_{N}\right) .
\end{aligned}
$$

Theorem 5.1. Let $\mathrm{S}=\mathrm{E}^{2}$, A be the family of right-angled triangles in $\mathrm{E}^{2}$ with sides containing the right angle parallel to coordinate axes. Then for arbitrary $\varepsilon>0$ if $N$ is large enough

$$
c_{1} N^{1 / 4-\varepsilon}<\Delta_{N}(A)<N^{1 / 4} \sqrt{\operatorname{Tog} N} \text {. }
$$

(For the lower bound see Schmidt (1969), for the upper bound Beck (1983 a)) Remark. If $A$ is the family of aligned rectangles, then

$$
c_{1} \log N<\Delta_{N}(A)<c_{2} \log N
$$

(See Theorem 4.2.) Compare the two results. There exists a set of $N$ 
points in the unit square such that the discrepancy in every aligned rectangle is not larger than $c \log N$. However, there must exist an aligned rectangle such that splitting it into two right-angled triangles, both will have a discrepancy as large as $1^{\frac{1}{4}-\varepsilon}$ (but of course of different signs).

These results, and actually all the other ones below raise the problem on which properties of the family $A$ does it depend whether the discrepancy is "large" or "sma11" $\left(N^{\alpha}\right.$ or $\left.(\log N)^{\beta}\right)$. The situation is rather annoying, we do not have a complete understanding of the problem.

A result related to the above one is given in the following theorem.

Theorem 5.2. Let $\mathrm{S}=\mathrm{E}^{2}$, A be the family of tilted rectangles in the plane (not necessarily contained in the unit square $E^{2}$ ). Then

$$
c_{1} N^{\frac{1}{4}-\varepsilon}<\Delta_{N}(A)<c_{2} N^{\frac{1}{4}} \cdot \sqrt{\log N} .
$$

The lower bound was proved by Schmidt (1969), the upper bound by Beck (1981 a).

A generalization for higher dimensions is the following theorem.

Theorem 5.3. (Beck (1983)). Let $U_{N}=\left\{u_{1}, \ldots, u_{N}\right\}$ be a set of $\mathbb{N}$ points on the $\mathrm{k}$-dimensional unit torus. Let $\mathrm{A}$ be the set of tirted squares with diameter at most 1 . Then

$$
N^{\frac{1}{2}-\frac{1}{2 k}} \sqrt{\log N}>\Delta_{N}(A)>c(d) N^{\frac{1}{2}-\frac{1}{2 k}} .
$$

Remark. Schmidt proved for $k=2,3$ the slightly weaker resuit that $\Delta_{N}(A)>N^{\frac{1}{2}-\frac{1}{2 k}-\varepsilon}$.

Theorem 5.4. (Schmidt (1969 b)). $S=\mathrm{E}^{\mathrm{k}}$, A be the family of balls in $\mathrm{R}^{\mathrm{k}}$ (not necessarily contained in $\mathrm{E}^{\mathrm{k}}$ ). Then

$$
N^{\frac{1}{2}-\frac{1}{2 k}}<\Delta_{N}(A)<C_{1} N^{\frac{1}{2}-\frac{1}{2 k}} \sqrt{\log N}
$$


Answering an old problem of Roth, very recently Beck proved the following theorem.

Theorem 5.5. (Beck (1983)). Let $S$ be the unit disc, $B$ the set of halfplanes, $A=\{S \cap B ; B \in B\}$. Then

$$
c_{1} N^{\frac{1}{4}-\varepsilon}(\log N)^{-1}<\Delta_{N}(A)<N^{\frac{1}{4}+\varepsilon} \log N .
$$

Theorem 5.6. (Beck (1983e)). Let $\left(u_{n}\right)$ be an infinite sequence of points in the plane. Then for every real number $r$ there exists a tizted square $A_{r}$ of side $r$, such that

$$
r^{\frac{1}{2}} \log r>\left|Z\left(A_{r}\right)-r^{2}\right|>c \cdot r^{\frac{1}{4}} \text {. }
$$

(Here $Z\left(A_{r}\right)$ denotes the number of points $u_{i} \in A_{r}$. )

This theorem is especially remarkable since this is the first one where a set of arbitrary size and of large discrepancy can be guaranteed.

$$
\begin{aligned}
& \text { For a set } U_{N}=\left\{u_{1}, \ldots, u_{N}\right\} \subset S \text { let } \\
& G=\left\{g \mid g: U_{N} \rightarrow\{+1,-1\}\right\} .
\end{aligned}
$$

Set

$$
D\left(A ; U_{N}\right)=\min _{g} \sup _{A \in A}\left|\sum_{x \in A \cap U_{N}} g(x)\right|
$$

and

$$
D_{N}(A)=\sup _{U_{N}} D\left(A ; U_{N}\right)
$$

G. Tusnady asked the order of magnitude of (or bounds for) $D_{N}(A)$ in the special case when $S=E^{2}$ and $A$ is the family of aligned rectangles. Recently Beck investigated this problem, for other families $A$ too.

Theorem 5.7. (Beck (1981 b)). Let $\mathrm{S}=\mathrm{E}^{2}, \mathrm{U}_{\mathrm{N}} \subset \mathrm{E}^{2}, \mathrm{~A}$ be the family of aligned rectangles. Then 


$$
c_{1} \log N<D_{N}(A)<c_{2}(\log N)^{4} .
$$

Again, for tilted rectangle the discrepancy is much larger.

Theorem 5.8. (Beck (1981 b)). Let $\mathrm{S}=\mathrm{E}^{2}, \mathrm{U}_{\mathrm{n}} \subset \mathrm{E}^{2}, \mathrm{~A}$ be the family of tilted rectangles. Then

$$
c_{1} N^{\frac{1}{4}-\varepsilon}<D_{N}(A)<c_{2} N^{\frac{1}{2}+\varepsilon} .
$$

Theorem 5.9. (Beck (1983 a)). Let $\mathrm{S}$ be the $\mathrm{N} \times \mathrm{N}$ Zattice, $\mathrm{A}$ be the family of tilted rectangles in the $\mathrm{N} \times \mathrm{N}$ lattice. Then

$$
c_{1} N^{\frac{1}{3}-\varepsilon}<D(A ; S)<N^{\frac{1}{2}} \sqrt{\log N} .
$$

If tilted rectangles not necessarily contained in the $N \times N$ square belong to $A$ too, the discrepancy is even much larger.

Theorem 5.10. (Beck $(1983 \mathrm{c})$ ). Let $\mathrm{S}$ be the $\mathrm{N} \times \mathrm{N}$ lattice, $A$ be the family of tilted rectangles (not necessarily contained in the $\mathrm{N} \times \mathrm{N}$ (attice). Then

$$
c_{1} N^{\frac{1}{2}}(\log N)^{-\frac{1}{2}}<D(A, S)<c_{2} N^{\frac{1}{2}}(\log N)^{\frac{1}{2}} .
$$

\section{$6(\{n \alpha\})-S E Q U E N C E S$ AND ERGODIC THEORY}

Most of the recent results for the distribution of point sets refer to the $d$-dimensional space for $d \geq 2$ and they have a definite geometric character. However, there is widely developed theory of uniformly distributed sequences in $[0,1)$ and only few of these results have been generalized for higher dimension.

We formulate all the results below for distributions of infinite sequences in $[0,1]$. According to the observation of Roth actually we could formulate the assertions for finite point-sets in $E^{2}$ as well.

The most important class of uniformly distributed sequences in $[0,1)$ is the class of sequences $\left(\left\{n_{\alpha}\right\}\right)$ for $\alpha$ irrational. These are the basic sequences in the theory of diophantine approximation. Further, these are the best "test-sequences": very often theorems which 
were found first for sequences $(\{n \alpha\})$ turned out to be true for more general ones. Finally we mention the relation of sequences ( $\{n\}\}$ ) to topological transformations.

The discrepancy of $\left(\left\{n_{\alpha}\right\}\right)$ depends on the partial quotients $a_{k}, k=1, \ldots$ of $\alpha$. For every $N$ and $x \in[0,1)$ there is an "explicit" formula for $\Delta_{N}([0, x)$ ) (see T. Sós (1974)). This leads e.g., to the following

Theorem 6.1. Let $\frac{p_{k}}{q_{k}}$ be the $k$-th convergent of $\alpha$; $\frac{p_{k}}{q_{k}}=\left[a_{1}, \ldots, a_{k-1}\right]$. If $q_{k} \leqq N<q_{k+1}$ then

$$
c_{1} \sum_{i=1}^{k} a_{i}<\max _{1 \leqq n \leq N} \Delta_{n}<c_{2} \sum_{i=1}^{k+1} a_{i} .
$$

Consequentzy, if $a_{i} \leqq K, i=1, \ldots$, then

$$
\Delta_{N}<c_{K} \log N
$$

Much is known about the finer properties of the distribution.

Though

$$
\max _{I \leqq n \leqq N} \sup _{I} \Delta_{n}(I)>c \log N \text {, }
$$

there are intervals $I$ in which the distribution is very good.

Theorem 6.2. For the sequence $(\{n \alpha\})$ and for a fixed inter-

val I

$$
\sup _{N} \Delta_{N}(I)<\infty,
$$

iff $|I|=\{k \alpha\}$ for some integer $k$.

The "if" part was proved by Hecke (1922) and much deeper "only if" part by Kesten (1966). Very elegant proofs and generalizations of this theorem in the framework of ergodic theory are due to Furstenberg, Keynes \& Shapiro (1973), Halász (1976), Petersen (1973).

On the other hand it is remarkable that this theorem (and further properties of $\Delta_{N}$ ) has consequences for ergodic theory (see e.g. 
Herman (1976 a,b), Delighe (1975).

Schmidt investigated the analogous question for arbitrary sequences in $[0,1)$.

Theorem 6.3. (Schmidt (1974)). For an arbitrary sequence $\left(u_{n}\right)$ in $[0,1)$ the lengths of all intervals I with $\sup _{N} \Delta_{N}(I)<\infty$ form at most a countable set.

The ergodic theoretical generalization shows the essence of Kesten's theorem. Let $(\Omega, A, \mu)$ be a probability space, $T: \Omega \rightarrow \Omega$ an ergodic transformation. For an $A \in A$ let $Z_{N}(A ; x)$ denote the number of points $T^{n} x \in A .1 \leqq n \leq N$. Set

$$
\Delta_{N}(A ; x)=Z_{N}(A ; x)-N_{\mu}(A)
$$

By Birkhoff's ergodic theorem, for fixed $A \in A$ for almost all $x \in \Omega$

$$
\frac{1}{N} \Delta_{N}(A ; X) \rightarrow 0 \text {, if } N \rightarrow \infty \text {. }
$$

Furstenberg, Keynes \& Shapiro (1973), Petersen (1973), Halasz (1976) proved the following generalization of Kesten's theorem:

Theorem 6.4. If for $A \in A \sup _{N}\left|\Delta_{N}(A ; x)\right|$ is bounded on a set $x \subset \Omega$ of positive measure, then $e^{2 \pi i \mu(A)}$ is an eigenvalue of $T$; i.e., there exists a function $g \neq 0$ such that

$$
g(T x)=e^{2 \pi i \mu(A)} g(x) \text { for } x \in \Omega
$$

On the other hand, for every eigenvalue $\mathrm{e}^{2 \pi \boldsymbol{i} \mu}$ there exists an $A \in A$ such that $\mu(A)=\mu$ and $\Delta_{N}(A ; x)$ is bounded in $N$ for almost alz $x \in \Omega$.

Remark. Kesten's theorem follows from the above one. To see this let $\Omega=R / \mathbb{Z}$. Let $\mu$ denote the Lebesgue-measure, $R_{\alpha}: x \rightarrow x+\alpha$ $\left(R_{\alpha}\right.$ is the rotation by $\left.\alpha 2 \pi\right)$. The eigenvalues of $R_{a}$ are the numbers $e^{2 \pi i\{k \alpha\}}$ which implies Kesten's theorem.

We give another example of the relationship between uniform distribution and ergodic theory. This illustrates how the results for distribution of the sequences ( $\{n \alpha\}$ ) imply general results for homeomorphisms of the circle. 
Denjoy (1932) proved that for every homeomorphism $T: R / \mathbb{R}+$ $\rightarrow \mathbf{R} / \mathbf{Z}$ having no periodic point there exists an irrational $\alpha(T) \in(0,1)$ such that $T$ is conjugate to the rotation $R_{\alpha}: x \rightarrow x+\alpha$. By this, if we consider (the orbit of a point $x \in R / \mathbb{Z}$ ) the distribution of $T^{n} x ; n=1, \ldots$, this is determined by the distribution of the sequence $(\{n \alpha\})$. E.g.,

(a) Let $Z_{N}(I ; x)$ denote the number of points $T^{n} x \in I$, $1 \leq n \leq N$ and $\mu$ the invariant measure belonging to $T$. By Birkhoff's ergodic theorem the remainder term $\Delta_{N}(I ; x)=Z_{N}(I ; x)-N_{\mu}(I)=0$ (N). quence $(\{n \alpha(T)\})$.

By Denjoy's theorem $\Delta_{N}(I ; x)$ is the same as that of the se-

(b) The order of points $\{n \alpha\}, 1 \leq n \leq N$ is very much restricted: if $\pi$ is the permutation determined by $\{\pi(1) \alpha\}<\ldots<\{\pi(N) \alpha\}$, then for example for every $\alpha$ and $N$ fixed $\pi(i)-\pi(i-1)$ takes at most three different values. Now, by Denjoy's theorem the same holds for an arbitrary homeomorphism $T$ and $x$ and permutations $\pi$ defined by $T^{\pi(1)}(x)<$ $<T^{\pi(2)}(x)<\ldots<T^{\pi(N)}$. (See T. Sos (1957), Swierczkowski (1958).)

One of the most fascinating and deepest relationships between combinatorics and ergodic theory is given by Furstenberg \& al.: Proof and generalization of Szemeredi's theorem. Since there is a recent expository paper by Furstenberg, Katznelson ornstein (1982), and the book of furstenberg (1981), we do not go into the discussion of this.

\section{Strong irregularity}

In $[0,1)$ the following "strong irregularity" phenomenon

holds.

\section{Theorem 6.5.}

A. For every $\varepsilon>0$ there exists $a \quad \delta>0$ (depending only on $\varepsilon$ ) such that given an arbitrary sequence $\left(u_{n}\right)$ in $(0,1)$

$$
\Delta_{n}>\delta \log n
$$

for all but at most $\mathrm{N}^{\varepsilon}$ values of $\mathrm{n} \leqq \mathrm{N}$.

B. For every $K$ there exists a $\delta$ (depending only on $K$ )

such that

$$
\Delta_{n}>K
$$

for all but at most $(\log N)^{\delta}$ values of $n \leqq N$. 
C. For an arbitrary sequence $\left(u_{n}\right)$ in $(0,1)$ the set of values of $x$ for which

$$
\Delta_{N}([0, x))=0(\log N)
$$

holds has Hausiorff dimension 0 .

This theorem was proved first only for $\left(\left\{n_{\alpha}\right\}\right)$ sequences (T. Sos (1979), (1983)), then for arbitrary sequences and in a more general form by Halasz (1981) and Tijdeman \& Wagner (1980).

One-sided irregularities

Measuring the irregularities with $\left\|\Delta_{N}\right\|_{\infty}$ or $\left\|\Delta_{N}\right\|_{p}$, we do not have any information on the sign of the discrepancy. In $\S 1$ we introduced the one-sided discrepancies $\Delta_{\mathrm{N}}^{+}$and $\Delta_{\mathrm{N}}^{-}$. The behaviour of $\Delta_{N}^{+}$and $\Delta_{N}^{-}$show some new phenomena.

Again, as almost always, the first results on one-sided irregularities were found for $\left(\left\{n_{\alpha}\right\}\right)$ sequences:

There is no one-sided strong irregularity phenomenon. We mention just the simplest illustrations of this. It is easy to see that

$$
\sup _{N} \Delta_{N}^{+}=\infty, \inf _{N}^{-} \Delta_{N}^{-}=\infty
$$

However, for an arbitrary sequence $M_{N}+\infty$ there exists an a such that

$$
\Delta_{N}^{+}<M_{N} \text { if } N>N_{0}
$$

(resp. there exists an a such that $\Delta_{N}^{-}<M_{N}$ ).

Similarly, it is easy to see that

$$
i_{N}^{+}<K
$$

can hold only for a sequence $\left(N_{k}\right)$ of 0 -density. However, for an arbitrary sequence $M_{N}=O(N)$, there exist an $\alpha$ and a $K$ such that

$$
\Delta_{n}^{+}<k
$$

holds for at most $M_{N}$ values of $n \leq N$, if $N>N_{0}$ (T. Sós (1983)). 
Concerning intervals of small discrepancy, first we remark that

$$
\sup _{N} \Delta_{N}^{+}((0, B))<\infty
$$

may hold also in the case when $\beta \neq\{k \alpha\}$, i.e. when

$$
\sup _{N} \Delta_{N}([0, \beta))=\infty \text {. }
$$

In Dupain \& T. Sos (1978) the characterization of the intervals $(0, \beta)$ with

$$
\sup _{N} \Delta_{N}^{+}([0, \beta))<\infty
$$

is investigated. Here we mention just one of the new phenomena: there exists an a for which the set $\left\{\beta \mid \sup _{N}^{+}([0, \beta))<\infty\right\}$ is of power of continuum.

However, the assertion in Theorem remains true, if instead of boundedness of $\Delta_{N}(A)$ we suppose only one-sided boundedness. Halász (1976) proved that if

$$
\sup _{N} \Delta_{N}^{+}(A ; x)<\infty
$$

holds on a set $X \subset \Omega$ of positive measure, then $e^{2 \pi i \mu(A)}$ must be an eigenvalue of $T$.

\section{PARTITION-PROBLEMS FOR GENERAL HYPFEGRAPHS}

While in the previous sections we had to make a strong selection because of the large variety and wide scope of the theorems, here we have only limited number of results. Though the problems for general hypergraphs arise from investigations of irregularities on different structures, they are interesting on their own too. To start with, we have to remark that there are almost no lower bounds on the discrepancy of partitions of hypergraphs.

The theorems below give upper bounds for the discrepancy of partitions on hypergraphs. One of the first results of this type is due to 01 son \& Spencer. 
We mention in advance that the results here are related to integer valued programming. The relation between discrepancy of sequences and convex programming was discussed already in Niederreiter (1972).

Theorem 7.1. (Ozson \& Spencer (1978)). Let $A=\left\{A_{1}, \ldots, A_{k}\right\}$ be a family of subsets of $S,|S|=n$. Consider the two-colorings of $S: G=\{g \mid g: S \rightarrow\{+1,-1\}\}$. Set

$$
\Delta(A)=\min _{g \in G} \max _{A \in A}\left|\sum_{x \in A} g(x)\right|
$$

and

$$
f(n ; k)=\max _{A} \Delta(A),
$$

where the maximum is extended over all families $A$ for which $A \mid=k$ and $\cup A=S$. Then $A \in A$

$$
f(n ; k)<((n+1) \log 2 k)^{1 / 2}
$$

Theorem 7.2. (olson \& Spencer (1978)). With the above nota-

tion set

$$
h(k)=\max _{A} \Delta(A)
$$

where the maximum is extended over all families $A$ for which $|A|=k$ Then

$$
\left(\frac{1}{2}-o(1)\right) k^{1 / 2}<h(k)<c k^{1 / 2} \log k
$$

if a Hadomard matrix of order $k+1$ exists.

The upper bound of Theorem 7.2 was improved by Beck \& Fiala.

Theorem 7.3. (Beck \& Fiala (1981)).

$$
h(k)<(2 k)^{1 / 2} \sqrt{\log 2 k}
$$

where $\mathrm{c}$ is a positive absolute constant. 
In most of the partition problems we can easily find a system of 1 inear equations describing the problem and having a fractional solution. How close we can get with integer values to the solution measures how large the discrepancy is.

The theorem of Beck \& Fiala below (generalizing a famous result of Baranyai (1975)) is used to get upper bounds for discrepancies.

Theorem 7.4. (Beck \& Fiala (1981)). Integer-making lemma. Let $\alpha_{1}, \ldots, \alpha_{s}$ be given real numbers. Let $A$ be a family of subsets of the index set $\{1, \ldots, s\}$ such that every $i \in\{1, \ldots, s\}$ belongs to at most $t$ members of $A$. Then there exist integers $a_{1}, \ldots, a_{s}$ so that $\left|a_{i}-\alpha_{i}\right|<1,1 \leq i \leq s$ and

$$
\left|\sum_{i \in A} a_{i}-\sum_{i \in A} \alpha_{i}\right| \leqq t-1 \text { for every } A \in A \text {. }
$$

Theorem 7.5. (Beck \& Fiala (1981)). Let d denote the maximal degree of the hypergraph $(S, A)$ :

$$
d(A)=\max _{x \subset S}|\{A \subset A ; x \subset A\}| .
$$

With the notations of Theorem 5.1:

$$
\Delta(A) \leq 2 d(A)-2
$$

Conjecture. (Beck \& Fiala). For arbitrary $(S, A)$

$$
\Delta(A) \leqq c(d(A) \log d(A))^{1 / 2}
$$

Theorem 7.6. (Beck (1981 a)). With the above notations

$$
\Delta(A)<c(d(A))^{1 / 2}(\log |A|)^{1 / 2} \log |S| .
$$

Remark. Beck (1981 a) used the above theorems e.g. to prove that Roth's estimate for the discrepancy of arithmetic progressions is nearly sharp. This application itself justifies that the theorem is valuable.

However, it is plausible that upper and lower bounds for 
$\Delta(A)$ depending on some structural properties e.g. on some intersection properties of the system $A$ should be found.

We do not know any general theorem for hypergraphs which gives a nontrivial lower bound for the discrepancy $\Delta(A)$.

01 son \& Spencer (1978) proved for a system A derived from the Hadamard matrix the discrepancy is greater than $c|A|^{1 / 2}$. (An analysis of their proof gives a more general lower bound.)

8 SOME FURTHER PROBLEMS

We have seen that for different structures different types of discrepancy problems were investigated. Here we give a list of problems for generâl hypergraphs, implicitly classifying the discrepancy-problems.

Let $(S, A)$ by a hypergraph.

Problem of proportional representation.

Let $\alpha \in(0,1)$ be given. We call an $\mathrm{S}_{\alpha} \subset \mathrm{S}$ an $\alpha$-representative set if

$$
\| S_{\alpha} \cap A|-\alpha| A \mid ! \leqq 1 \text { for every } A \in A \text {. }
$$

\section{Problem 1.}

Find conditions on the existence of an $\alpha$-representative system in the terms of the structure of the hypergraph.

(1) is a very strong requirement. When it cannot be satisfied, the question is how close can we get to it.

Problem 2.

Find bounds on

$$
\min _{S^{\prime} \subset S} \max _{A \in A}|| S^{\prime} \cap A|-\alpha| A||
$$

(or on

$$
\left.\min _{S^{\prime} \subset S}\left(\sum_{A \in A}|| S \cap A|-\alpha| A||^{p}\right)^{\frac{1}{p}} .\right)
$$

(Does there exist any min-max theorem for some special classes?)

The problems below are formulated for general hypergraphs.

Let $\alpha_{1}, \ldots, \alpha_{r} \in(0,1)$ be given and $\sum \alpha_{i}=1$. We say that 
$S=\sum_{i=1}^{r} S_{\alpha_{i}}, S_{\alpha_{i}} \cap S_{\alpha_{j}}=\emptyset$, ifj is an $\left(\alpha_{1}, \ldots, \alpha_{r}\right)$-partition

$$
|| S_{\alpha_{i}} \cap A\left|-\alpha_{i}\right| A|| \leqq 1
$$

for every $A \in A$ and $1 \leqq i \leqq r$.

\section{Problem 3.}

Find conditions on the existence of an $\left(\alpha_{1}, \ldots, \alpha_{r}\right)$-partition. Let $P(A) \in R^{r}$ denote the vector $\left(\left|S_{1} \cap A\right|-\alpha_{1}|A|, \ldots, S_{r} \cap A \mid-\alpha_{r} ; A_{1}\right)$.

Probiem 4.

Find bounds on

$$
S=\sum_{i=1}^{\min _{i} S_{i}} \max _{A \in A}\|P(A)\|
$$

where $\|$ it is a norm on $R^{r}$ (or on

$$
\left.S=\min _{i=1} S_{i}\left(\sum_{A \in A}|| S \cap A|-\alpha| A||^{P}\right)^{\frac{1}{p}}\right) \text {. }
$$

Problem of well distributed sequences.

Here we consider the "dynamical" version of the previous problem; we want to find a sequence $\left(u_{n}\right)$ in $S$ for which every segment is proportionally distributed.

Let $\left(u_{n}\right)$ be a finite resp. infinite sequence in $s$, and $u_{n}=\left\{u_{1}, \ldots, u_{n}\right\}$. We say that $\left(u_{n}\right)$ is uniformly distributed with respect to $A$ if

$$
\Delta_{n}(A)=|| U_{n} \cap A|-| \frac{A}{S}|n| \leq 1
$$

for every $n \leq N$ (resp. for every $n$ ) and for every $A \subset A$.

Problem 5.

Find conditions on the existence of a uniformly distributed sequence. 
Problem 6.

Find bounds on

$$
\min _{\left(u_{n}\right)} \max _{n \leqq N} \max _{A \in A} \Delta_{n}(A)
$$

Von Neumann in one of his first papers proved that any everywhere dense sequence in $(0,1)$ can be rearranged to a uniformly distributed sequence.

In the flavor of this simple theorem the following can be asked. We formulate it only in the finite case.

Problem 7.

Let $u_{i} \in S, \quad 1 \leq i \leq N$ be a sequence with discrepancy $\Delta_{N}$. For any permutation $\pi$ consider the sequence $\Delta_{n}^{\pi}, n=1, \ldots, N$ as the discrepancy of $u_{\pi(1)}, \ldots, u_{\pi(n)}$. Give bounds on

$$
\min _{\pi} \max _{n} \Delta_{n}^{\pi}
$$

or more generally, for a given function $\phi(n)$

$$
\min _{\pi} \max _{n} \phi(n) \Delta_{n}^{\pi} .
$$

On strong irregularity.

We mentioned in $\S 6$ the "strong irregularity" phenomenon for sequences in $(0,1)$. The analogous question can be asked for many other cases. (In this direction see also Theorem 5.6.)

on one-sided irregularity.

As explained in $\S 6$ new phenomena emerge if we consider the signed discrepancy $\left(\Delta_{n}^{+}, \Delta_{n}^{-}\right)$for $(\{n \alpha\})$ sequences. Instead of stating particular questions we just call the attention to this problem.

Decomposition problems.

Problem 1.

The hypergraph $(S, A)$ is called totally unimodular, if every $S^{\prime} \subset S$ has a partition $g: S^{\prime} \rightarrow\{+1,-1\}$ such that $\left|\sum_{x \in A} g(x)\right| \leqq 1$ if $A \in A$. (This is equivalent to the following: every square submatrix of the incidence matrix of $(S, A)$ has determinant 0 or \pm 1.$)$ 
Assume there is a partition $A=\cup_{i=1}^{d} A_{i}$ such that $\left(S, A_{i}\right)$,

$1 \leqq i \leqq d$ is totally unimodular. Is it true, that there exists a $K=K(d)$ depending only on $d$ such that $A$ has an $\alpha$-representative system of discrepancy $F(d)$ ?

Probzem 2.

Given $\Delta$ and $d$, does there exist a $\tilde{\Delta}=\tilde{\Delta}(\Delta, d)$ with the following property. If $A=\cup \mathcal{U}_{i=1}$ and the discrepancy of $\left(S, A_{i}\right), 1 \leqq i \leqq d$ is $\Delta$, then the discrepancy of $(S, A)$ is at most $\tilde{\Delta}$.

Added in proof.

Just after having finished this paper, J. Beck proved a surprising and deep theorem. This generalizes several results for families in $\mathrm{R}^{\mathrm{k}}$ and gives an answer to the important question, on which geometrical properties does the order of magnitude of the discrepancy depend.

Theorem. (Beck). Let $\mathrm{S}$ be a square of sides $\mathrm{N}$ in $\mathrm{R}^{2}$ anci let be given $\mathrm{N}^{2}$ arbitrary points in $\mathrm{S}$. Let $\mathrm{B}$ be a conver domain aru. $B(\lambda, \tau, \underline{V})$ the domain obtained from $B$ by a similarity transformation of dilation $\lambda \leqq 1$, of rotation $\tau$ and translation by $\underline{v} \in R^{2}$. Let $\mu(B)$ resp. $l(B)$ denote the area resp. the length of the circumference of $B$, and let $7(B(\lambda, \tau, \underline{v}))$ denote the number of points in $B(\lambda, \tau, \underline{v})$. Ther there exist $\lambda_{0}, r_{0}, \underline{v}_{0}$ such that

$$
\left|Z\left(B\left(\lambda_{0}, \tau_{0}, \underline{v}_{0}\right)\right)-\mu\left(B\left(\lambda_{0}, \tau_{0}, \underline{v}_{0}\right) \cap S\right)\right|>c \sqrt{\min (\mu(B), l(B))}
$$

where $c>0$ is an absolute constant.

Acknowledgement.

My thanks are due to J. Beck and M. Simonovits for their valuable comments on the paper and to $J$. Beck for informing me about his very recent results too. 
REFERENCES

Ajtai, M., Erdös, P., Komlōs, J. \& Szemerědi, E. (1981) : On Turăn's

theorem for sparse graphs. Combinatorica 1 (4) 313-317.

Ajtai, M., Komlós, J., Pintz, J., Spencer, J. \& Szemerëdi, E. (1983):

Extremal uncrowded hypergraphs. In manuscript.

Ajtai, M., Komlós, J. \& Szemerëdi, E. (1981) : A dense infinite Sidon

sequence. European Joumal of Combinatorics 2, 1-11.

Beck, J. (1981 a): Balancing families of integer sequences. Combinatorica

$1(3)$.

Beck, J. (1981 b): Balanced two-colorings of finite sets in the square.I.

Combinatorica 1 (4) 327-335.

Beck, J. (1981 c): Roth's estimate on discrepancy of integer sequences is

nearly sharp. Combinatorica 1 (4).

Beck, J. (1983 a): Some upper bounds in the theory of irregularities of

distribution. Acta Arithm. To appear.

Beck, J. (1983 b): Unpublished.

Beck, J. (1983 c): On a problem of K. F. Roth concerning irregularities

point distribution. To appear.

Beck, J. (1983 d): Irregularities of two-colorings of the integer coor-

dinate points in the plane. To appear.

Beck, J. \& Fiala, I. (1981): Integer making theorems. Discrete Applied Math. 3, 1-8.

Beck, J. \& Spencer, J. (1983): Well distributed 2-colorings of integers relative to long arithmetic progressions. To appear.

Bollobás, B. (1980): Exxtromal Graph Theory. Academic Press.

Bollobás, B. \& Erdbs, P. (1976): On a Ramsey-Turăn type problem. J. Comb. Th. Ser. B 21, 166-168.

Burki11, H. \& Mirsky, L. (1973): Monotonicity. J. Math. AnaZ. AppZ. 41, 391-410.

Davenport, H. (1956): Note on irregularities of distribution. Mathematika $3,131-135$.

Deligne, P. (1975): Les diffémorphitsmes du cercle. Springer, Lecture Notes in Mathematics Sẻminaire Bourbaki, 477, 01.

Denjoy, A. (1932): Sur les courbes dēfinies par des équations differentielles a la surfacedu tore. J. de Math. pures et appliquées $11,333-375$. 
Dupain, Y. \& T. Sós, V. (1978): On the one-sided boundedness of discrepancy function of the sequence (n $\alpha$ ). Acta Arithm. 37, 363-374.

ErdØs, P. (1964): Problems and results on diophantine approximations. Comp. Math. $16,52-65$.

Erdbs, P. (1967): Some recent results on extremal problems in graph theory. Theory of Graphs. Gordon \& Beach, New York, 117-130.

Erdbs, P., Hajnal, A., T. Sós, V. \& Szemerëdi, E. (1983): On results of Ramsey-Turan type theorems. Combinatorica. To appear.

Erdbs, P., Meir, A., T. Sŏs, V. \& Turăn, P. (1972 b): On some applications of' graph-theory III. Discrete Math. 2, 207-228.

Erdరbs, P., Meir, A., T. Sŏs, V. \& Turăn, P. (1972 a): On some applications of graph-theory II. Studies in Pure Math. Acad. Press, 89-100.

ErdOs, P., Meir, A., T. Sös, V. \& Turăn, P. (1972): On some applications of graph-theory I. Canadian Math. Buzz. 15 (1), 27-32.

ErdBs, P. \& Simonovits, M. (1966): A limit theorem in graph theory. Studia Sei. Math. Hungar. 1, 51-57.

ErdDs, P. \& T. Sós, V. (1969): Some remarks on Ramsey's and Turăn's theorem. Col1. Math. Soc. Bolyai 4 Combinatorial Theory, Balatonfuired, 395-404.

Erdðs, P. \& T. Sós, V. (1982): On Ramsey-Turăn type theorems for hypergraphs. Combinatorica 2 (3).

Erdys, P. \& Spencer, J. (1972): Imbalances in k-colorations. Networks 1 , 379-385.

Erdos, P. \& Stone, M. H. (1946): On the structure of linear graphs. Buzz. Amer. Math. Soc. 52, 1087-1091.

Erd8s, P. \& Szemeredi, E. (1972): On a Ramsey-type theorem. Period. Math. Hungar. 2, 295-299.

Fürstenberg, H. (1981): Recurrence in ergodic theory and combinatorial number theory. Princeton Univ. Press, Princeton, N. J.

Fürstenberg, H., Katznelson, Y. \& Ornstein, D. (1982): The ergodic theoretical proof of Szemeredi's theorem. Buzz. Amer. Math. Soc. 7 (3) 447-654.

Fürstenberg, H., Keynes, H. \& Shapiro, L. (1973): Prime flows in topological dynamics. Israel J. Math. 14 (1) 26-38.

Gallai, T. (1931): published in Rado (1933 a).

Gerencsër, L. \& Gyărfas, A. (1967): On Ramsey-type problems. Ann. Univ. Sci. Budapest. Eötvös sect. Math. 10, 167-170.

Graham, R. L., Rothschild, B. L. \& Spencer, J. H. (1980): Romsey theory. Wiley Interscience Series in Discrete Mathematics.

Graham, R. L. (1981): Rudiments of Ramsey theory. Regional conference series in mathematics, No. 45 .

Graham, R. L., Leeb. L. \& Rotnschild, B. L. (1972): Ramsey's theorem for a class of categories. Adv. Math. 8, 417-433. 
Halasz, G. (1976): Remarks on the remainder in Birkhoff's ergodic theorem. Acta Math. Hungar. 27, 389-396.

Halasz, G. (1981): On Roth's method in the theory of irregularities of point-distributions. Froc. Conf. Analytic Number Theory at Durham, 1979.

Hecke, E. (1922): Uber analytische Funktionen und die Verteilung von Zahlen mod Eins. Abh. Math. Sem. Hamburg 1, 54-76.

Herman, M. R. (1976): Conjugaison $c^{\infty}$ des difféomorphismes du cercle dont le nombre de rotation satisfait a une condition arithmętique. C. R. Acad. Sci. Paris 282.

Kesten, H. (1966/67): On a conjecture of ErdOs and Szüsz related to uniform distribution mod 1. Acta Axithn. 12, 193-212.

Koksma, J. (1942/43): Een algemeene stelling uit de theory der geli kmatige verdeeling modulo 1. Mathematica $B$ (Zutphen) 11, 7-11.

Komlös, J., Pintz, J. \& Szemerëdi, E. (1982): A lower bound for Heilbronn's problem. Journal of London Matt. Soc.

Kuipers, L. \& Niederreiter, H. (1974): Uniform distribution of sequences. Pure \& Aprizied Mathematics, Miley-Interscience.

Niederreiter, H. (1972): On the existence of uniformly distributed sequences in compact spaces. Compositio Math. 25, 93-99.

Niederreiter, H. (1978): Quasi-Monte-Carlo methods and pseudo-random numbers. Buzz. Amer. Math. Soc. 84, 957-1041.

Petersen, K. (1973): On a series of cosecants related to a problem in ergodic theory. Comp. Math. 26 (3) 313-317.

Rado, R. (1933 a): Verallgemeinerung eines Satzes von Van der Waerden mit Anwendungen auf ein Problem der Zahlentheorie. Sonderausg. Sitzungsber. Preuss. Akad. Wiss. Fhis. Math. Kilasse 17, 1-10.

Rado, R. (1943): Note on combinatorial analysis. Proc. Loridon Math. Soc. $48,122-160$.

Ramsey, F. P. (1930): On a problem of formal logic. Froc. London Math. Soc. 30, 264-286.

Roth, K. F. (1952): Sur quelques ensembles d'entiers. C. R. Acad. Sci. Paris 234, 388-390.

Roth, K. F. (1954): On irregularities of distribution. Mathematika 1, 7379.

Roth, K. F. (1964): Remark concerning integer sequences. Acta Arithm. 9, $257-260$. 
Roth, K. F. (1979): On irregularities of distribution III. Acta Aritron. $35,373-384$.

Roth, K. F. (1980): On irregularities of distribution IV. Acta Artirm. $37,67-75$.

Sårközy, A. (1972): See Erdbs-Spencer: Probabizistic Methods in Combinatorics Akadēmiai Kiadó, Budapest 1974.

Schmidt, W. M. (1969): Irregularities of distribution IV. Inv. Math. 7 $55-82$.

Schmidt, W. M. (1972): Irregularities of distribution VII. Acta Arithm. $21,45-50$.

Schmidt, W. M. (1974): Irregularities of distribution VIII. Trans. Amer. Math. Soc. 198, 1-22.

Schmidt, W. M. (1977): Lectures on Irregularities of Distribution. Tata Inst. of Fund. Res. (Bombay), Lectures on Math. and Phys. 56. Schur, I. (1916): Ober die Kongruenz $x^{m}+y^{m} \equiv z^{m} \bmod p$. Jber. Deutsch. Math. Verein. 25, 114-116.

Simonovits, M. (1968): On methods for solving extremal problems in graph theory stability problems. Theory of Graphs. Proc. Coll. held in Tihany, Academic Press, New York, 279-319.

T. Soss, V. (1957): On the theory of diophantine approximation I. Acta Math. Acad. Sci. Hungar. 8, 461-472.

T. Soss, V. (1958): On the distribution mod 1 of the sequence $\{n \alpha\}$. Ann. Univ. Sci. Budapest. Eötvös Sect. Math. 1, 127-134.

T. Sos, V. (1958): On the theory of diophantine approximation II. Acta Moth. Hungar. IX (1-2) 229-241.

T. Sõs, V. (1968): On a theorem of H. Kesten. Tagungsbericht Obemwolfach, Zahlentheorie, p. 18.

T. Sós, V. (1972): On the discrepancy of the sequence (na). Tagungsbericht Obemolfach, 28.

T. Soss, V. (1974): On the discrepancy of the sequence $(n \alpha)$. Colz. Math. Soc. J. Bolyai 13, 359-367.

T. Soss, V. (1969): On extremal problems in graph theory. Proc. Calgary Internat. Conf. on Comb. Structures, 407-410.

T. Soss, V. (1979): On strong irregularities of the distribution of $\{n \alpha\}$ sequences. Tagungsbericht Oberwolfach 23, 17-18.

T. Sŏs, V. (1983): Strong irregularities of the distribution of (na) sequences 1. Studies in Pure Math. Akadémiai Kiadó. (Turán memorial volume). 
T. Sos, V. (1983 b): Strong irregularities and one-sided discrepancy. (Under publication.)

Szemeredi, E. (1973): On graphs containing no complete subgraph with 4 vertices (in Hungarian). Mat. Lapok 23, 111-116.

Swierczkowski (1958): On successive settings of an arc on the circumference of a circle. Fund. Math. 46, 187-189.

Tijdeman, R. (1980): The chairman assignment problem. Discrete Math. 32 323-330.

Tijdeman, R. \& Wagner, G. (1980): A sequence has almost nowhere small discrepancy. Monatshefte Math. 90, 315-329.

Turån, P. (1941): Eine extremale Aufgabe aus der Graphentheorie (in Hungarian). Mat. Fiz. Lapok 48, 436-452. See also: On the theory of graphs. Colloquizm Math. 3, 19-30.

Van der Corput, J. G. (1935 a): Verteilungsfunktionen I. Proc. Kon. Ned. Akad. $v$. Wetensch. 38, 813-821.

Van der Corput, J. G. (1935 b): Verteilungsfunktionen II. Froc. Kon. Ned. Akad. v. Wetensch. 38, 1058-1066.

Van Aardenne Ehrenfest, T. (1945): Proof of the impossibility of a just distribution of an infinite sequence of points over an interval. Indag. Math. 7, 71-76.

Weyl, H. (1916): Ober die Gleichverteilung von Zahlen mod Eins. Math. Ann. 77, 313-352.

Van der Waerden, B. L. (1927): Beweis einer Bandetschen Vermutung, Nieiw Arch. Wisk. 1 


\section{ADDENDA AND ERRATA}

Certain works cited in the paper have been omitted from the references. They appear to be the following but there was insufficient time in which to confirm this with the author.

Baranyai, Z. (1975): On the factorization of the complete uniform hypergraph. Col1. Math. Soc. Bolyai 10. Infinite and finite sets, Keszthely, Vol I, 91-108.

Erdös, P. and Graham, R.L. (1980): $01 d$ and new problems and results in combinatorial number theory. Monographie de l'Enseignement Mathematique, No. 28.

Hales, A. and Jewett, R.I. (1963): Regularity and positional games. Trons. Amer. Math. Soc. 106, 222-229.

Herman, M. R. (1976b): Conjugaison $\mathrm{C}^{\infty}$ des diffemorphismes du cercle pour presque tout nombre de rotation. C.R. Acad. Sci. Paris 283.

01son, J. and Spencer, J.H. (1978): Balancing families of sets. J. Combin. Theory Ser. A 25, 29-37.

Rado, R. (1933b): Studien zur Kombinatorik. Math. Zeit. 36, 424-480.

Page 219, line 9. For "Erdös-Komlös-..." read "Ajtai-Komlös-..."(?)

E.K.L. 